\title{
Prevalencia y evaluación de síntomas en enfermedad renal crónica avanzada
}

\author{
Daniel Gutiérrez Sánchez ${ }^{1}$, Juan P. Leiva-Santos ${ }^{1}$, Rosa Sánchez-Hernández², Rafael Gómez García ${ }^{1}$ \\ ${ }^{1}$ Fundación CUDECA, Málaga, ${ }^{2}$ Servicio de Nefrología. Hospital General de Villalba, Madrid. España
}

\section{Resumen}

El paciente con enfermedad renal crónica avanzada (ERCA) presenta una elevada carga de síntomas que contribuyen a un aumento del sufrimiento y disminuyen su calidad de vida. El uso de instrumentos de evaluación es esencial para el control de síntomas. El objetivo de esta revisión es obtener una visión contrastada de los instrumentos más usados para evaluar síntomas en ERCA, realizando una descripción de la prevalencia de síntomas en esta población. Método: Se realizó una revisión de la literatura publicada sobre estudios en los que se utilizara algún instrumento para medir la intensidad de varios síntomas en pacientes con ERCA. La búsqueda se realizó en Pubmed, Cochrane, SciELO, TESEO, PROQOLID y BiblioPRO. Los criterios de inclusión fueron: estudios realizados con pacientes en ERCA, que evaluaran la sintomatología con algún instrumento de medición de síntomas, y que además, indicaran la prevalencia de varios síntomas. Resultados: Los instrumentos identificados fueron el Memorial Symptom Assessment Scale Short Form(MSAS-SF), el Dialysis Symptom Index (DSI), el Edmonton Symptom Assessment System (ESAS) y el Palliative care Outcome Scale-Symptoms Renal (POS-S RENAL). En pacientes adultos con ERCA sometidos a tratamiento renal sustitutivo con hemodiálisis y diálisis peritoneal, los síntomas más prevalentes fueron el cansancio, prurito, estreñimiento, anorexia, dolor, alteraciones del sueño, ansiedad, disnea, nauseas, piernas inquietas, y depresión. Éstos síntomas fueron similares en pacientes con manejo renal conservador, y presentaron un patrón común con la sintomatología de otras enfermedades avanzadas. Concluimos que existe necesidad de investi-

\footnotetext{
Correspondencia:

Daniel Gutiérrez Sánchez

Fundación Cudeca
}

Av. del Cosmos. 29631 Arrollo de la Miel. Málaga

Email: danieltunie@hotmail.com gar sobre prevalencia y evaluación de síntomas en esta población, y que el uso sistemático de los instrumentos específicos de evaluación de síntomas como medida de resultados es fundamental.

\author{
PALABRAS CLAVE \\ - PREVALENCIA DE SÍNTOMAS \\ - ENFERMEDAD RENAL CRÓNICA AVANZADA \\ - INSTRUMENTOS DE EVALUACIÓN DE SÍNTOMAS \\ - CUIDADOS PALIATIVOS RENALES
}

\section{Prevalence and evaluation of symptoms in advanced chronic kidney disease}

\begin{abstract}
The patient with advanced chronic kidney disease (ACKD) has a high symptom burden that contribute to increased suffering and diminish their quality of life. The use of symptom assessment tools is essential for the control of symptoms. The aim of this review is to obtain a contrasted vision of the instruments commonly used to assess symptoms in $A C K D$, making a description of the prevalence of symptoms in this population. Method: A review of the literature on studies in which an instrument is used to measure the intensity of several symptoms in patients with ACKD was undertaken. The search was conducted in PubMed, Cochrane, SciELO and TESE0. Inclusion criteria were: studies in patients with ACKD, evaluating symptoms with an assessment tool, and also indicate the prevalence of various symptoms. Results: The instruments identified were the Memorial Symptom Assessment Scale Short Form (MSAS-SF), the Dialysis Symptom
\end{abstract}


Index (DSI), the Edmonton Symptom Assessment System (ESAS) and the Palliative Care Outcome Scale-Symptoms Kidney (POS-S RENAL). In adult patients with $A C K D$ undergoing renal replacement therapy with hemodialysis and peritoneal dialysis, the most prevalent symptoms were fatigue, pruritus, constipation, anorexia, pain, sleep disturbance, anxiety, dyspnea, nausea, restless legs, and depression. These symptoms were similar in patients with renal conservative management, and showed a common pattern to the symptoms of others advanced diseases. We conclude that we need to research about the prevalence and evaluation of symptoms in this population, and a systematic use of specific instruments for evaluating symptoms as an outcome measure is necessary.

\section{KEYWORDS}

- PREVALENCE OF SYMPTOMS

- ADVANCED CHRONIC KIDNEY DISEASE

- SYMPTOMS ASSESSMENT TOOLS

- RENAL PALLIATIVE CARE

\section{Introducción}

El aumento de las enfermedades crónicas es uno de los cambios más significativos del perfil epidemiológico mundial del siglo XXI la enfermedad renal crónica (ERC) representa un problema de salud pública, cuya incidencia y prevalencia es elevada, siendo pacientes con elevada morbimortalidad. Según las guías K/DOQI (Kidney Disease Outcome Quality Initiative) y KDIG0 2012 (Kidney Disease Improving Global Outcomes) ${ }^{3,4}$, se define ERC (independientemente del diagnóstico clínico) como la presencia durante al menos tres meses de un filtrado glomerular (FG) inferior a $60 \mathrm{ml} /$ min/1'73 m² o lesión renal (definida por la presencia de anormalidades estructurales o funcionales del riñón, que puedan provocar potencialmente un descenso del FG. Según las guías mencionadas anteriormente, y atendiendo al FG, la ERC se clasifica en diferentes estadios:

- Estadio 1. Daño renal con $F G \geq 90$ o FG normal

- Estadio 2. Daño renal con FG 60-89 o ligeramente disminuido

- Estadio 3. FG moderadamente disminuido 30-59

- Estadio 4. FG gravemente disminuido 15-29

- Estadio 5. Fallo renal < 15, ERCA
Cuando la ERC avanza, requiere tratamiento renal sustitutivo (TRS) con hemodiálisis (HD), diálisis peritoneal (DP), trasplante renal (TXR) o bien manejo renal conservador (MRC). La ERCA afecta sobre todo a la población de mayor edad, incrementándose el riesgo con los años ${ }^{5,6}$.

El paciente con ERC podría beneficiarse de cuidados paliativos (CP) desde el diagnóstico de la enfermedad, durante las etapas de TRS, en el cese de TRS y especialmente si se decide MRC. Se considera que el MRC es una opción conveniente para pacientes con alta comorbilidad, edad avanzada y que no quieran iniciar diálisis. Cada vez se documenta un mayor número de pacientes que eligen esta modalidad de tratamiento ${ }^{7}$.

El futuro apunta a integrar los principios y prácticas de los CP en áreas de Nefrología, reconociéndose su aplicación como un índice de calidad en la atención de las enfermedades crónicas ${ }^{8-9,10}$. En nuestra cultura existe un interés creciente por los CP aplicados a la nefrología.

Debido a que los síntomas no controlados al final de la vida contribuyen a un mayor sufrimiento, su tratamiento en fases avanzadas de la enfermedad es una prioridad $^{11}$. Los síntomas emocionales como la ansiedad y la depresión se asocian a mayores niveles de síntomas somáticos ${ }^{12}$. Además, la calidad de vida relacionada con la salud (CVRS) en pacientes con ERCA es peor que la de la población de referencia en las dimensiones estudiadas, y varía según el tipo de tratamiento que reciba el paciente, la edad y sexo $0^{13,14}$. De hecho, los síntomas percibidos por estos pacientes están relacionados con una peor CVRS ${ }^{15,16}$. En este sentido, la colaboración de los profesionales pertenecientes a las áreas de la nefrología y de cuidados paliativos, puede influir positivamente en la calidad de vida del paciente y la familia ${ }^{17}$.

El control de síntomas constituye un objetivo asistencial, y por tanto, es fundamental realizar una valoración exhaustiva del número e intensidad de síntomas que sufre el paciente renal. La medición de síntomas como referencia subjetiva, no es fácil, pero resulta fundamental por lo que, cada vez es más necesario disponer de instrumentos de medida de síntomas adaptados a patologías específicas que faciliten la medición y que puedan utilizarse en la práctica clínica y en la investigación. El objetivo de esta revisión es obtener una visión contrastada de los instrumentos más usados para evaluar síntomas en ERCA, realizando una descripción de la prevalencia de síntomas en esta población. 


\section{Objetivos}

- Objetivo Principal: Describir los instrumentos usados para evaluar síntomas en pacientes con ERCA.

- Objetivo Secundario: Describir los síntomas más prevalentes en ERCA según las diferentes modalidades de tratamiento.

\section{Metodología}

Se realizó una revisión de la literatura publicada sobre estudios que hubieran utilizado algún instrumento para medir la prevalencia de varios síntomas en pacientes con ERCA tanto en MRC como en HD y DP.

La búsqueda se realizó en las bases de datos de Pubmed, Cochrane, Embase, SciELO y TESEO, utilizando como estrategias de búsqueda los términos previamente consultados en MESH: "outcome assessment", "symptoms assessment tool", "scale development", "questionnaires" "symptoms", "prevalence of symptoms" "chronic kidney disease", "advanced renal disease", "dialysis", "renal conservative management" "end of life" $y$ "palliative care", haciendo uso de los operadores booleanos "AND/OR". Esta estrategia de búsqueda se complementó con la revisión de la bibliografía de los artículos identificados.

Los criterios de inclusión fueron: estudios realizados con pacientes en ERCA en MRC, HD o DP, que evaluaran la sintomatología con algún instrumento de medición, y que además, indicaran la prevalencia de varios síntomas.

La búsqueda bibliográfica se realizó entre los meses de Octubre de 2014 y Junio de 2015.

\section{Resultados}

Se identificaron 276 artículos utilizando las estrategias de búsqueda mencionadas, y de ellos se revisaron
86. Solo 13 artículos reunían los criterios de inclusión ${ }^{18-25,28-30,32,33}$.

\section{Prevalencia de síntomas en ERCA}

\section{Prevalencia de Síntomas en Tratamiento Renal Sustitutivo: HD y DP}

Estudios realizados en pacientes adultos con ERCA sometidos a TRS con HD y DP, muestran como estos enfermos sufren un amplio número de síntomas. Concretamente, los síntomas más prevalentes son el cansancio, prurito, estreñimiento, anorexia, dolor, alteraciones del sueño, ansiedad, disnea, náuseas, piernas inquietas, y depresión, evidenciándose como más de la mitad de pacientes en diálisis sufren síntomas como el dolor, cansancio, picor o estreñimiento ${ }^{18}$. Además se describen otro tipo de síntomas menos prevalentes como calambres musculares y boca seca. Los resultados se describen en la tabla 1.

En esta línea, un estudio realizado al respecto en nuestra cultura mostró como entre los síntomas más frecuentes en enfermos en HD, se encontraban el cansancio, los picores, la piel seca, la sed, dolores de huesos y articulaciones y alteraciones del sueño, síntomas que fueron más frecuentes y severos en mujeres y en pacientes con ansiedad y depresión, mostrando como estos estados de ansiedad y depresión afectaban a estos pacientes en un $24 \%$, en el caso de la ansiedad y casi en un $50 \%$ en el caso de la depresión ${ }^{19}$.

\section{Prevalencia de Síntomas en MRC}

En pacientes adultos con ERCA en MRC, también podemos encontrar una amplia variedad de síntomas cuya frecuencia es similar a los de los pacientes en TRS (Figura 1).

Los síntomas más prevalentes en pacientes en MRC, son la debilidad, falta de energía, poco apetito, prurito, som-

Tabla 1. Prevalencia de síntomas ERCA. Hemodiálisis y diálisis peritoneal ${ }^{18}$

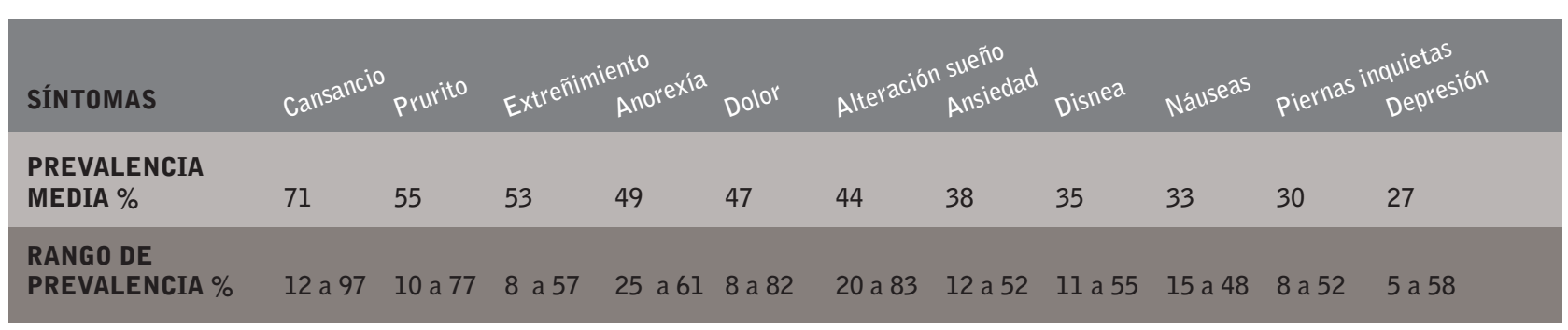




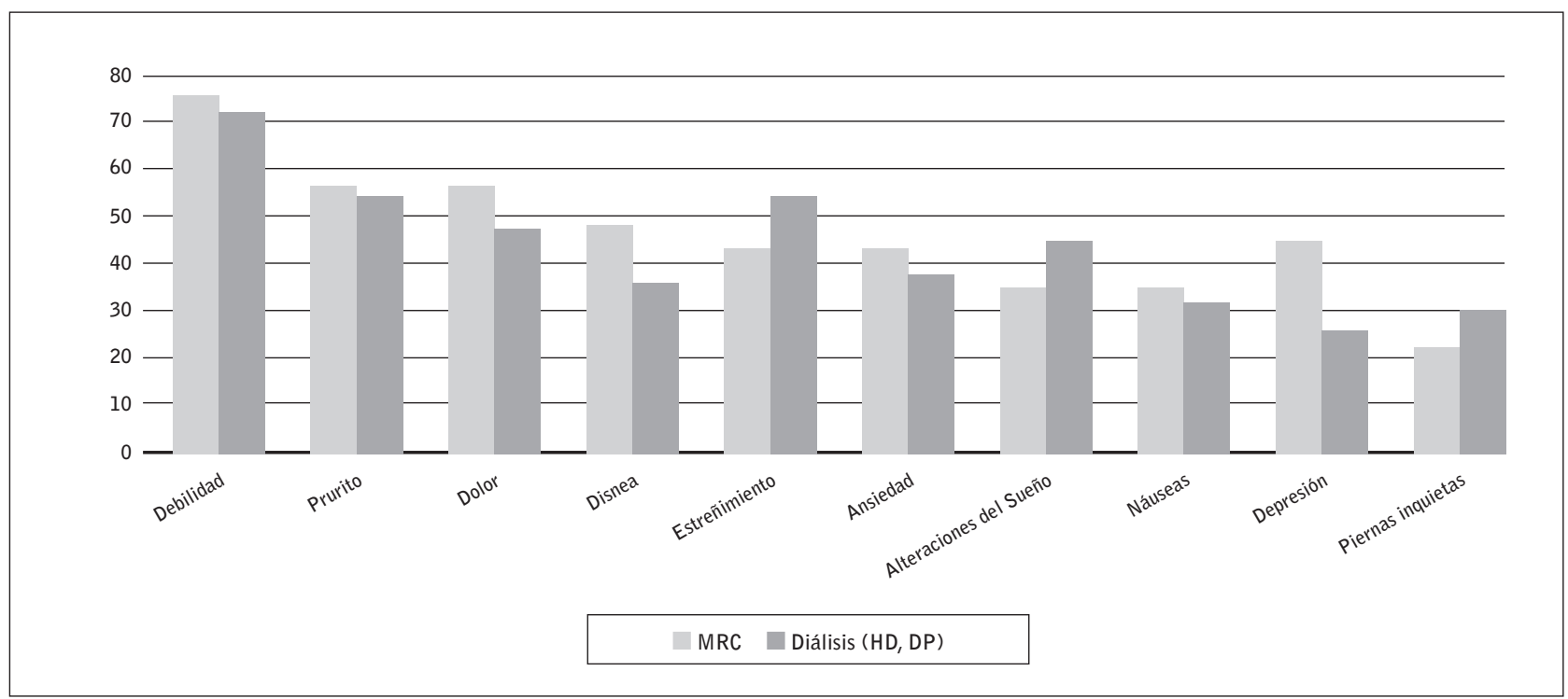

Figura 1. Prevalencia de Síntomas en ERCA. MRC 22 y Diálisis (HD, DP). ${ }^{18}$

nolencia, disnea, dolor, edema, y dificultad para dormir ${ }^{20}$. Además, según otros estudios realizados el estreñimiento, alteraciones en la piel y boca seca son también frecuentes (Tabla 2) 21,22. También podemos observar como en estados muy avanzados de ERCA (un mes antes del fallecimiento del paciente), aumenta la intensidad y frecuencia de síntomas como la debilidad, somnolencia, prurito y disnea (Tabla 2$)^{23}$.

\section{Prevalencia de síntomas en ERCA y otras enferme- dades crónicas avanzadas}

La carga de síntomas en ERCA es alta y similar a la producida por otras enfermedades crónicas avanzadas. Si comparamos los síntomas más prevalentes en ERCA con otras enfermedades crónicas avanzadas como IC, EPOC, SIDA y cáncer, podemos ver como el $50 \%$ de los pacientes en ERCA y otras enfermedades presentan dolor, disnea y cansancio ${ }^{24}$. En este sentido, cabe destacar el insomnio y la anorexia como síntomas recurrentes en todas estas patologías, encontrándose similitud en la prevalencia de un total de 11 síntomas (Tabla 3) ${ }^{24}$. De manera similar, en una revisión sistemática donde se describen los síntomas en ERCA y otras enfermedades por fallo orgánico en estadios avanzados como IC y EPOC, se demostró que los síntomas más prevalentes en estas tres enfermedades producidas por fallo de órgano eran similares, indicando que síntomas como el cansancio, la disnea, el dolor o el insomnio son frecuentemente percibidos por pacientes que sufren estas patologías, siendo el cansancio, el síntoma más frecuentemente experimentado ${ }^{25}$. Por tanto, también en este estudio se encontraron similitudes en lo que a prevalencia de síntomas se refiere para estas tres patologías 25 .

\section{Instrumentos para la evaluación de síntomas en ERCA}

Entre los instrumentos más utilizados para el estudio de síntomas en pacientes renales se han identificado una serie de cuestionarios no específicos para enfermos renales, como el Memorial Symptom Assessment Scale Short Form (MSAS-SF) ${ }^{26}$ y el Edmonton Symptom Assessment System (ESAS) ${ }^{33}$, y otros que han sido diseñados para medir síntomas en ERCA, como el Dialysis Symptom Index (DSI) ${ }^{30}$ y el Palliative care Outcome Scale-Symptoms Renal (POS-S Renal) ${ }^{21}$. Además en la literatura podemos encontrar estudios que han usado la dimensión de síntomas de cuestionarios que miden CVRS en el enfermo renal como el Kidney Disease Questionnaire, para la medición de síntomas ${ }^{19}$. A continuación se describen los instrumentos utilizados para la evaluación de síntomas en pacientes renales.

\section{Memorial Symptom Assessment Scale Short Form (MSAS-SF)}

EI MSAS-SF es la versión corta del MSAS, un instrumento de evaluación de síntomas que fue desarrollado para población oncológica ${ }^{26}$. Este cuestionario se ha usado para medir síntomas en diferentes grupos de pacientes, entre ellos los pacientes renales ${ }^{27,28}$. Es un cuestionario que puede ser cumplimentado por el propio paciente, en el cual se pregunta al paciente 
[ Daniel Gutiérrez Sánchez, et al ]

Prevalencia y evaluación de síntomas en enfermedad renal crónica avanzada

Tabla 2. Prevalencia de síntomas en ERCA. MRC..$^{21,22,23}$

\begin{tabular}{|c|c|c|c|c|c|c|}
\hline \multirow{2}{*}{ SÍNTOMAS } & \multicolumn{2}{|c|}{$\begin{array}{l}\text { Murphy et al., } 2009.22 \\
\qquad N=55\end{array}$} & \multicolumn{2}{|c|}{$\begin{array}{l}\text { Murtagh et al., } 2007.23 \\
\qquad N=66\end{array}$} & \multicolumn{2}{|c|}{$\begin{array}{c}\text { Murtagh et al., } 2010 .{ }^{24} \\
\text { Un mes antes de fallecer. } \mathrm{N}=49\end{array}$} \\
\hline & $\begin{array}{l}\text { PREVALENCIA } \\
\text { MEDIA } \%\end{array}$ & $\begin{array}{c}\text { RANGO DE } \\
\text { PREVALENCIA } \\
\%\end{array}$ & $\begin{array}{l}\text { PREVALENCIA } \\
\text { MEDIA \% }\end{array}$ & $\begin{array}{c}\text { RANGO DE } \\
\text { PREVALENCIA } \\
\%\end{array}$ & $\begin{array}{l}\text { PREVALENCIA } \\
\text { MEDIA } \%\end{array}$ & $\begin{array}{c}\text { RANGO DE } \\
\text { PREVALENCIA } \\
\%\end{array}$ \\
\hline Debilidad & 75 & $61-85$ & 76 & $66-84$ & 86 & $73-94$ \\
\hline Poca Movilidad & 75 & $61-85$ & $N R^{*}$ & NR & NR & NR \\
\hline Apetito Pobre & 58 & $44-71$ & 47 & $37-58$ & 71 & $57-83$ \\
\hline Edema & NR & NR & 58 & $47-66$ & 71 & $57-83$ \\
\hline Prurito & 56 & $42-70$ & 74 & $65-82$ & 84 & $70-93$ \\
\hline Dolor & 56 & $42-70$ & 53 & $42-63$ & 73 & $59-85$ \\
\hline Calambres musculares & NR & NR & 50 & $39-60$ & NR & NR \\
\hline Somnolencia & 49 & $35-63$ & 65 & $54-74$ & 82 & $68-91$ \\
\hline Disnea & 49 & $35-63$ & 61 & $50-70$ & 80 & $66-90$ \\
\hline Falta de concentración & NR & NR & 44 & $34-54$ & 76 & $61-87$ \\
\hline Estreñimiento & 42 & $29-56$ & 35 & $26-45$ & 65 & $50-78$ \\
\hline Ansiedad & 42 & $29-56$ & NR & NR & NR & NR \\
\hline Alteraciones Del sueño & 36 & $24-50$ & 41 & $32-51$ & NR & NR \\
\hline Náuseas & 36 & $24-50$ & NR & NR & 59 & $44-73$ \\
\hline Cambios en la piel/piel seca & 35 & $22-49$ & 42 & $32-53$ & NR & NR \\
\hline Depresión & 33 & $21-47$ & NR & NR & NR & NR \\
\hline Vómitos & 25 & $15-39$ & NR & NR & NR & NR \\
\hline Piernas inquietas & 24 & $13-37$ & 48 & $38-58$ & NR & NR \\
\hline Problemas bucales/boca sec & 20 & $10-33$ & 50 & $39-60$ & 69 & $55-82$ \\
\hline Diarrea & 11 & $4-22$ & NR & NR & NR & NR \\
\hline
\end{tabular}

*NR: Datos no recogidos.

Tabla 3. Síntomas ERCA, Cáncer, SIDA, IC y EPOC. ${ }^{24}$

\begin{tabular}{|c|c|c|c|c|c|}
\hline SÍNTOMA & ERCA & CÁNCER & SIDA & IC & EPOC \\
\hline Dolor & $47-50 \% . N=370$ & $35-96 \% . N=10.379$ & $63-80 \% . N=942$ & $41-77 \% . N=882$ & $34-77 \% . N=372$ \\
\hline Depresión & $5-60 \% . N=956$ & $3-77 \% . N=4.378$ & $10-82 \% . N=616$ & $9-36 \% . N=80$ & $37-71 \% . N=150$ \\
\hline Ansiedad & $39-70 \% . N=72$ & $13-79 \% . N=3.274$ & $8-34 \% . N=346$ & $49 \% . N=80$ & $51-75 \% . N=1.008$ \\
\hline Confusión & $N R^{*}$ & $6-93 \% . N=9.154$ & $30-65 \%$. NR & $18-32 \% . N=343$ & $18-33 \% . N=309$ \\
\hline Astenia & $73-87 \% . N=116$ & $32-90 \% . N=2.888$ & $54-85 \% . N=1.435$ & $69-82 \% . N=409$ & $68-80 \% . N=285$ \\
\hline Disnea & $11-62 \% . N=334$ & $10-70 \% . N=10.029$ & $11-62 \% . N=504$ & $60-88 \% . N=948$ & $90-95 \% . N=372$ \\
\hline Insomnio & $31-71 \% . N=351$ & $9-69 \% . N=5.606$ & $74 \% . N=504$ & $36-48 \% . N=146$ & $55-65 \% . N=150$ \\
\hline Náuseas & $30-43 \% . N=362$ & $6-68 \% . N=9.140$ & $43-49 \% . N=689$ & $17-48 \% . N=146$ & NR \\
\hline Estreñimiento & $29-70 \% . N=483$ & $23-65 \% . N=7.602$ & $34-35 \% . N=689$ & $38-42 \% . \mathrm{N}=80$ & $27-44 \% . N=150$ \\
\hline Diarrea & $21 \% . N=19$ & $3-29 \% . N=3.392$ & $30-90 \% . N=504$ & $12 \% . N=80$ & NR \\
\hline Anorexia & $25-64 \% . N=395$ & $30-92 \% . N=9.113$ & $51 \% . N=504$ & $21-41 \% . N=146$ & $35-67 \% . N=150$ \\
\hline
\end{tabular}

*NR: Datos no recogidos. 
los síntomas que ha padecido durante la última semana así como la intensidad de los síntomas que ha percibido. Está formado por 32 ítems con formato de respuesta tipo Likert de 5 puntos, donde el sujeto asigna la intensidad de cada uno de los síntomas a una categoría que oscila desde "nada" $(0)$ hasta "muchísimo" (4), para los 28 primeros síntomas. Para los cuatro últimos síntomas, el rango de intensidad oscila desde "rara vez" (1) hasta "casi constantemente" (4), obteniendo puntuaciones más elevadas cuando la intensidad es mayor. Además el paciente puede añadir otros síntomas percibidos que no figuren en el cuestionario. Este instrumento está formado por tres subescalas: síntomas físicos, psicológicos e índice de distrés global, y ha sido traducido y adaptado al españo ${ }^{29}$. EI MSAS-SF es un instrumento válido y fiable, que ha demostrado tener sensibilidad al cambio clínico del paciente, lo cual nos permite monitorizar síntomas y evaluar intervenciones ${ }^{29}$.

\section{Dialysis Symptom Index (DSI)}

EI DSI es un instrumento que ha sido desarrollado a partir del MSAS-SF, para su empleo en pacientes en HD. Este cuestionario autoadministrado pregunta al paciente que síntomas ha padecido durante la última semana y consta de 30 ítems medidos con formato de respuestas tipo Likert de 5 puntos, con un rango de intensidad para cada síntoma que oscila desde "nada" (0) hasta "muchísimo" (4), siendo el grado de intensidad más elevado a mayor puntuación ${ }^{30}$. Además el paciente puede añadir otros síntomas percibidos que no figuren en el cuestionario. Este instrumento no ha sido traducido al español y solo ha sido validado para su uso en pacientes en HD.

\section{Edmonton Symptom Assessment System (ESAS)}

EI ESAS es un instrumento que evalúa 10 síntomas con una escala numérica de intensidad que oscila del 0 al 10, siendo mayor la intensidad con puntuaciones más altas.

Ha sido adaptado y traducido a la cultura española y su uso está recomendado para evaluar síntomas en pacientes oncológicos en estadios avanzados ${ }^{31}$. Su versión modificada para uso en pacientes con ERCA, ha sido utilizada en otras culturas para evaluar síntomas en este tipo de pacientes ${ }^{32}$.

Es una herramienta fiable y adecuada para la evaluación de síntomas, que en su versión española ha demostrado tener adecuadas propiedades psicométricas.

\section{Palliative care Outcome Scale-Symptoms Renal (POS-S Renal)}

EI POS-S Renal es un instrumento para la evaluación de síntomas, que ha demostrado su utilidad en la evaluación de síntomas en pacientes con ERCA ${ }^{21,33}$. Es un autocuestionario de rápida y fácil cumplimentación que ha sido desarrollado para evaluar síntomas en ERCA. Está formado por 17 síntomas con formato de respuesta tipo Likert de 5 puntos, donde el paciente asigna el cómo le han afectado cada uno de los síntomas a una categoría que oscila desde "nada" $(0)$ hasta "insoportable"(4), aumentando la intensidad con puntuaciones más elevadas. Esta herramienta pregunta al paciente sobre síntomas percibidos durante la última semana y además permite añadir otros síntomas que no figuren en el cuestionario.

Este instrumento ha sido desarrollado por el equipo del "King's College Of London" y aún no ha sido adaptado ni traducido a ningún idioma.

\section{Discusión}

Desde estadios iniciales de la ERCA, los síntomas que la acompañan son muy variados y frecuentes, afectando a la esfera física y emocional del paciente de manera similar en las diferentes modalidades de tratamiento. En la sintomatología del paciente renal influyen varios factores como el propio fallo renal (prurito y síndrome de piernas inquietas), la comorbilidad asociada (neuropatía diabética, angina, etc.) o los factores relacionados con el propio TRS (calambres, problemas para dormir relacionados con alarmas nocturnas en DP) ${ }^{34}$. Sin embargo, cuando comparamos la prevalencia de síntomas en MRC y TRS, no encontramos diferencias significativas, aunque según los diferentes estudios realizados sobre pronóstico en pacientes en MRC y pacientes en TRS, los pacientes en TRS presentan un mayor número de ingresos hospitalarios, así como un mayor número de procedimientos diagnósticos y terapéuticos agresivos, y que disminuyen el número de días libres de hospital. En este sentido, el MRC es una opción que debe ser considerada, ya que en pacientes con edad avanzada y elevada comorbilidad, el TRS no siempre ofrece ventajas en lo que supervivencia y carga de síntomas se refiere ${ }^{35-36,37}$. Por eso, actualmente existe una tendencia creciente hacia la elección de MRC sobre Diálisis por parte de los pacientes ${ }^{38}$. Los escasos estudios realizados en España, impiden comparar el rango de síntomas padecidos en las diferentes modalidades de tratamiento, y por tanto es necesario investigar y profundizar en este aspecto. 
La diferencia en la trayectoria de la enfermedad, siendo más fluctuante en el caso de las enfermedades por fallo de órgano y SIDA, es una de las principales razones por la que existe un escaso desarrollo de programas asistenciales para pacientes paliativos no oncológicos. Estas diferencias de pronóstico sugieren que los cuidados paliativos deben de estar disponibles desde fases tempranas de la ERCA y el inicio de la atención por equipos multidisciplinares pertenecientes al ámbito de los cuidados paliativos, debe basarse en otros aspectos como el control de síntomas más que en el pronóstico.

Una de las limitaciones de esta revisión es la gran heterogeneidad de los estudios incluidos. Esta heterogeneidad es debida principalmente a la variedad de diseños de estudios, las diferencias en el método de recogida de datos y uso de diferentes cuestionarios para recoger información y la variabilidad del tamaño muestral. Aún así los datos se muestran en forma de rango de prevalencia con máximo y mínimo.

Cuando analizamos los resultados de los estudios sobre sintomatología en ERCA y otras enfermedades avanzadas, podemos observar como existe un patrón sintomatológico común al final de la vida, lo que indica que la atención paliativa en estas enfermedades puede ser relevante. Por tanto, es necesario invertir más esfuerzo en la evaluación y control de síntomas como el dolor, la disnea, el cansancio o el insomnio, que son comunes y afectan a un elevado número de pacientes con enfermedades avanzadas. En este sentido, el uso de instrumentos específicos es útil en la evaluación de síntomas y monitorización de los resultados de la asistencia en estos pacientes y por eso es necesario profundizar en el estudio de los síntomas en ERCA y su evaluación tanto en la práctica clínica como en investigación.

Llama la atención como la mayor parte de los estudios realizados sobre prevalencia de síntomas en ERCA, utilizan instrumentos de evaluación de síntomas genéricos y que no han sido diseñados para medir síntomas en esta población.

En comparación con los instrumentos genéricos, el POS-S Renal ha sido desarrollado para evaluar síntomas en ERCA, y por eso incluye síntomas prevalentes en el paciente renal como el picor, síndrome de piernas inquietas, estreñimiento y diarrea. Además de la posibilidad de poder ser cumplimentado por el propio paciente, el uso de la escala de categoría tipo likert, permite obtener una menor ambigüedad de respuestas ${ }^{39}$. No hemos encontrado ningún cuestionario específico y que permita medir la intensidad de varios síntomas en
ERCA en nuestra cultura, y por eso, es necesario adaptar instrumentos como el POS-S Renal.

\section{Conclusiones}

Existe una necesidad importante de investigar sobre la incidencia y prevalencia de síntomas en ERCA, así como evaluación de síntomas y de las intervenciones que permitan avanzar en el control de síntomas en esta población. La evaluación estandarizada de síntomas y la monitorización de los resultados de tratamiento en estos pacientes es fundamental.

La intervención de equipos multidisciplinares pertenecientes al ámbito de la nefrología y cuidados paliativos puede representar una ventaja significativa en el control sintomático y en el alivio del sufrimiento de los pacientes y sus familias.

\section{Conflicto de interés}

Los autores declaran que no tienen conflicto de interés potencial.

\author{
Recibido: 27 agosto 2015 \\ Revisado: 29 agosto 2015 \\ Modificado: 29 agosto 2015 \\ Aceptado: 30 agosto 2015
}




\section{Bibliografía}

1. Alcázar R, Egocheaga MI, Orte L et al. [SEN-SEMFYC consensus documento chronic kidney disease]. Nefrologia 2008;28(3):273-282.

2. Otero A, de FA, Gayoso P, Garcia F. Prevalence of chronic renal disease in Spain: results of the EPIRCE study. Nefrologia 2010;30(1):78-86.

3. K/DOQI clinical practice guidelines for chronic kidney disease: evaluation, classification, and stratification. Am J KidneyDis 2002; 39(2 Suppl(1):S1-266.

4. KDIG0 2012 Clinical Practice Guideline for the Evaluation and Management of Chronic Kidney Disease. KidneyInt (Suppl) 2013;3(1):1-308.

5. Sociedad Española de Nefrología: Registros de diálisis y trasplante 2007. (Fecha de acceso Abril 2014). Disponible en: http://www.senefro.org/modules. php? name $=$ webstructure \&id webstructure $=128$

6. Sociedad Española de Nefrología: Registros de diálisis y trasplante 2012. (Fecha de acceso Abril 2014). Disponible en: http://www.senefro.org/modules. php? name $=$ webstructure \&idwebstructure $=128$

7. $0^{\prime}$ Connor NR, Kumar P. Conservative management of end-stage renal disease without dialysis: a systematic review. J PalliatMed. 2012 Feb;15(2):228-35.

8. Brown, Mark A., Susan M. Crail, Rosemary Masterson, CelineFoote, Jennifer Robins, IvorKatz, Elizabeth Josland, et al. ANZSN Renal Supportive Care Guidelines 2013.Nephrology(Carlton, Vic.) 18, no. 6 (2013): 401-454.

9. Leiva-Santos JP, Sánchez-Hernández R, García-Llana $\mathrm{H}$, Fernández-Reyes MJ, Heras-Benito M, Molina-Ordas Á, et al. Cuidados de soporte renal y cuidados paliativos renales: revisión y propuesta en terapia renal sustitutiva. Nefrología 2012 Jan;32(1):20-7.

10. Callahan D. Death and the research imperative. $\mathrm{N}$ Engl J Med 2000;342:654-6.

11. Steinhauser KE, Christakis NA, Clipp EC, McNeilly M, McIntyre L, Tulsky JA. Factors considered important at the end of life by patients, family, physicians, and other care providers. JAMA. 2000;284(19):2476-82.

12. Perales-Montilla CM, Duschek $S$, Reyes-Del Paso $\mathrm{GA}$. The influence of emotional factors on the report of somatic symptoms in patients on chronic haemo- dialysis: the importance of anxiety. Nefrologia. 2013 Nov 13;33(6):816-25.

13. Goma S, Anna; Peris A P y RAMOS ALCARIO,A B. Calidad de vida en pacientes con insuficiencia renal crónica en tratamiento con diálisis. Nefrología 2010; 13,155-160.

14. Rodríguez Fructuoso, M. et al. Calidad de vida en la enfermedad renal crónica. Nefrología. 2011;31:9196.

15. Attalya Frank MSW, Gail K. Auslander DSW, MPH \& Joshua Weissgarten MD (2004) Quality of Life of Patients with End-Stage Renal Disease at Various Stages of the Illness, Social Work in Health Care, 38:2, 1-27.

16. Abdel-Kader K, Unruh ML, Weisbord SD. Symptom burden, depression, and quality of life in chronic and end-stage kidney disease. Clinical journal of the American Society of Nephrology : CJASN. 2009;4(6):1057-64.

17. Guía para el tratamiento conservador en pacientes con Enfermedad Renal Crónica Avanzada, Govern de les Illes Balears, 2015. (Fecha de acceso Abril 2014). Disponible en: http://www.caib.es/sacmicrofront/archivopub.do?ctrl...id=190069

18. Murtagh FEM, Addington-Hall J, Higginson IJ. The prevalence of symptoms in end-stage renal disease: a systematic review. Advances in chronic kidney disease. 2007;14(1):82-99

19. Álvarez-Ude F, Fernández-Reyes MJ, Vázquez A, Mon C, Sánchez R, Rebollo P: Síntomas físicos y trastornos emocionales en pacientes en programa de hemodiálisis periódica. Nefrología 21: 191-199, 2001.

20. $0^{\prime}$ Connor NR, Kumar P. Conservative management of end-stage renal disease without dialysis: a systematic review. J Palliat Med. 2012 Feb;15(2):228-35.

21. Murphy EL, Murtagh FEM, Carey I, Sheerin NS. Understanding symptoms in patients with advanced chronic kidney disease managed without dialysis: use of a short patient-completed assessment tool. Nephron Clinical practice. 2009;111(1):c74-c80.

22. Murtagh FEM, Addington-Hall JM, Edmonds PM, Donohoe P, Carey I, Jenkins K, et al. Symptoms in advanced renal disease: a cross-sectionalsurvey of symptom prevalence in stage 5 chronic kidney disease managed without dialysis. J PalliatMed. 2007;10(6):1266-76. 
23. Murtagh FE, Addington-Hall J, Edmonds $P$, Donohoe $P$, Carey I, Jenkins K, et al. Symptoms in the month before death for stage 5 chronic kidney disease patients managed without dialysis. Journal of pain and symptom management. 2010;40(3):342-52.

24. Solano JP, Gomes B, Higginson IJ. A comparison of symptom prevalence in far advanced cancer, AIDS, heart disease, chronic obstructive pulmonary disease and renal disease. Journal of pain and symptom management. 2006;31(1):58-69.

25. Janssen DJA, Spruit MA, Wouters EFM, Schols JMGA. Daily symptom burden in end-stage chronic organ failure: a systematic review. PalliatMed. 2008;22(8):938-48.

26. Portenoy RK, Thaler HT, Kornblith AB, Lepore JM, Friedlander-Klar $\mathrm{H}$, Kiyasu $\mathrm{E}$, et al. The Memorial Symptom Assessment Scale: an instrument for the evaluation of symptom prevalence, characteristics and distress. European journal of cancer (0xford, England : 1990). 1994;30A(9):1326-36.

27. Vogl D, Rosenfeld B, Breitbart W, Thaler H, Passik $S$, McDonald M, et al. Symptom prevalence, characteristics, and distress in AIDS outpatients. Journal of pain and symptom management. 1999;18(4):25362.

28. Weisbord SD, Carmody SS, Bruns FJ, Rotondi AJ, Cohen LM, Zeidel ML, et al. Symptom burden, quality of life, advance care planning and the potential value of palliative care in severely ill haemodialysis patients. Nephrol Dial Transplant. 2003 Jul;18(7):1345-52.

29. Dapueto JJ, Abreu M del C, Francolino C, Levin R. Psychometric assessment of the MSAS-SF and the FACIT-Fatigue Scale in Spanish-speaking patients with cancer in Uruguay. Journal of pain and symptom management. 2014;47(5):936-45.

30. Weisbord SD, Fried LF, Arnold RM, Rotondi AJ, Fine MJ, Levenson DJ, et al. Development of a symptom assessment instrument for chronic hemodialysis patients: the Dialysis Symptom Index. J Pain Symptom Manage. 2004 Mar;27(3):226-40.

31. Carvajal A, Hribernik N, Duarte E, Sanz-Rubiales A, Centeno C. The Spanish Version of the Edmonton Symptom Assessment System-Revised (ESAS-r): First Psychometric Analysis Involving Patients With Advanced Cancer. Journal of Pain and Symptom Management. 2013 Jan;45(1):129-36.
32. Davison SN, Jhangri GS, Johnson JA. Cross-sectional validity of a modified Edmonton symptom assessment system in dialysis patients: a simple assessment of symptom burden. KidneyInt. 2006;69(9):1621-5.

33. Brennan F, Collett $G$, Josland EA, Brown MA. The symptoms of patients with CKD stage 5 managed without dialysis. Progress in Palliative Care [Internet]. 2014 Dec 8 [cited 2015 May 21]; Available from: http://www.maneyonline.com/doi/abs/10.1179/1743 291X14Y.0000000118

34. Brown MA, Crail SM, Masterson R, Foote C, Robins J, Katz I, et al. ANZSN renal supportive care 2013: opinion pieces [corrected]. Nephrology (Carlton). 2013 Jun;18(6):401-54.

35. Foote C, Ninomiya T, Gallagher M, Perkovic V, Cass A, McDonald SP, et al. Survival of elderly dialysis patients is predicted by both patient and practice characteristics. Nephrology, dialysis, transplantation : official publication of the European Dialysis and Transplant Association - European Renal Association. 2012;27(9):3581-7.

36. Carson RC, Juszczak M, Davenport A, Burns A. Is maximum conservative management an equivalent treatment option to dialysis for elderly patients with significant comorbid disease? Clinical journal of the American Society of Nephrology : CJASN. 2009;4(10):1611-9.

37. Hussain JA, Mooney A, Russon L. Comparison of survival analysis and palliative care involvement in patients aged over 70 years choosing conservative management or renal replacement therapy in advanced chronic kidney disease. Palliative Medicine. 2013;27(9):829-39.

38. Morton RL, Snelling P, Webster AC, Rose J, Masterson $R$, Johnson DW, et al. Factors influencing patient choice of dialysis versus conservative care to treat end-stage kidney disease. Canadian Medical Association Journal. 2012;184(5):E277-83.

39. Cañadas Osinski I, Sánchez Bruno A. Categorías de respuesta en escalas tipo Likert. Psicothema. 10(3):623-31. 